CERN-TH.6721/92

ACT-22/92

CTP-TAMU-75/92

UMN-TH-1117/92

\title{
FLIPPED HEAVY NEUTRINOS: FROM THE SOLAR NEUTRINO PROBLEM TO BARYOGENESIS
}

\author{
John Ellis \\ Theoretical Physics Division, CERN \\ CH - 1211 Geneva 23 \\ and \\ D.V. Nanopoulos \\ Center for Theoretical Physics, Department of Physics, Texas A\&M University \\ College Station, TX 7r843-4242, USA \\ and \\ Astroparticle Physics Group, Houston Advanced Research Center (HARC) \\ The Woodlands, TX 77382, USA \\ and
}

Keith A. Olive

School of Physics and Astronomy, University of Minnesota

Minneapolis, MN 55455, USA

\begin{abstract}
We discuss baryogenesis using the flipped $S U(5)$ model for lepton mass matrices. We show that the generalized see-saw mechanism in this model can not only provide MSW neutrino mixing suitable for solving the solar neutrino problem, and supply a hot dark matter candidate $\left(\nu_{\tau}\right)$ with mass $0(10) \mathrm{eV}$ as indicated by recent COBE results, but can also naturally account for the baryon asymmetry of the universe. Heavy singlet neutrino decay generates a net lepton asymmetry which is subsequently reprocessed by nonperturbative electroweak interactions. We evaluate the baryon asymmetry so produced in light of the constraints that the COBE observations put on inflationary cosmologies, finding it comfortably consistent with observation.
\end{abstract}

CERN-TH.6721/92

ACT-22/92

CTP-TAMU-75/92

UMN-TH-1117/92

November 1992 

Cosmology, astrophysics and particle physics have for many years been in constant and fruitful interaction. Much of this was triggered by the advent of Grand Unified Theories (GUTs), which predicted novel phenomena that had the potential to solve many of the most important problems in cosmology, and were perhaps easier to test in astrophysics than in the laboratory. Characteristic examples of these phenomena were the baryon-number-violating interactions that could have been responsible for the baryonto-entropy ratio $n_{B} / s$, and the lepton-number-violating interactions leading to neutrino masses that could help explain the apparent solar neutrino deficit and the Dark Matter in the Universe.

The initial euphoria in astroparticle physics was somewhat dampened when these GUT miracles were subjected to closer inspection. For example, it has been realized that non-perturbative effects in the Standard Model violate baryon and lepton number conservation [1], could have erased a GUT-generated baryon asymmetry [2], and might even be able to generate the baryon-to entropy ratio themselves [3]. Moreover, for some time the solar neutrino story did not settle down [4], and hot neutrino Dark Matter became disfavoured by theorists of galaxy formation [5].

The balance of optimism has now been somewhat redressed. The realization is increasing that non-perturbative baryon-number-violating interactions have a limited ability to erase a GUT baryon or lepton asymmetry [6, 7], and as yet no demonstrated ability to generate a baryon-to-entropy ratio of the observed magnitude [3] Moreover, recent observations of the solar neutrinos seem to confirm that there is indeed a solar neutrino deficit that is difficult to explain by astrophysics alone [8], and the recent COBE [9] observations of large-scale density perturbations tend to favour models with a mixture of hot and cold Dark Matter.

The closest approximation to the traditional GUT framework that is permitted by the usual string model-building techniques is flipped $S U(5)$ [10, 11]. We are therefore motivated to revisit these familiar baryon- and lepton-number-violating astroparticle interfaces in the context of this model. Indeed, two of us (J.E and D.V.N.) together with J. Lopez [12] pointed out recently that flipped $S U(5)$ ties together in a natural way the Mikheyev-Smirnov-Wolfenstein (MSW) [13] interpretation of the apparent solar neutrino deficit and the $\mathrm{O}(10) \mathrm{eV}$ neutrino favoured by COBE and other measurements of largescale structure, and suggests that $\nu_{\mu}-\nu_{\tau}$ oscillations could be observable in forthcoming accelerator neutrino experiments. In this paper we extend the previous analysis to include an elegant mechanism for baryogenesis, modelled on the non-GUT heavy neutrino decay mechanism utilizing sphaleron interactions discussed by Fukugita and Yanagida [14].

The central role in our analysis is played by the massive singlet neutrino that is an unavoidable facet of flipped $S U(5)$. It leads to a see-saw neutrino mass matrix 11 , 15, 16, 17] that accommodates naturall solar, COBE and other data. We argue here that its decays naturally provide a lepton asymmetry which non-perturbative Standard Model interactions recycle into the observed baryon-to-entropy ratio. Heavy neutrino decays had been discussed previously 18 as a possible source of the baryon asymmetry, but before the realization of the important implications of Standard Model baryon-number-violating interactions. Also predating this realization were previous analyses of baryogenesis in flipped $S U(5)$ 19, which were, frankly, rather complicated and yielded asymmetries of marginal magnitude. Here we demonstrate explicitly, using our previous flipped neutrino 
analysis and the latest weak interaction parameters [12], and allowing for reheating of the Universe after inflation at the scale indicated by the COBE density perturbations [20], that decays of the massive flipped neutrino can explain naturally the observed baryon-toentropy ratio.

Before starting our analysis, we first remind the reader of the superpotential of the minimal flipped $S U(5)$ model, and introduce our notation. The renormalizable terms in the superpotential are [11]

$W=\lambda_{1 i j} F_{i} F_{j} h+\lambda_{2 i j} F_{i} \bar{f}_{j} \bar{h}+\lambda_{3 i j} \bar{f}_{i} l_{j}^{c} h+\lambda_{4} H H h+\lambda_{5} \bar{H} \bar{H} \bar{h}+\lambda_{6 i j} F_{i} \bar{H} \phi_{j}+\lambda_{7 i} h \bar{h} \phi_{i}+\lambda_{8 i j k} \phi_{i} \phi_{j} \phi_{k}$

where the $F_{i}, \bar{f}_{i}$ are $\mathbf{1 0}$ - and $\overline{\mathbf{5}}$-dimensional matte representations of SU(5), the $H$ and $\bar{H}$ are $\mathbf{1 0}$ and $\overline{\mathbf{1 0}}$ representations, the $h$ and $\bar{h}$ are $\mathbf{5}$ and $\overline{\mathbf{5}}$ Higgs representations, and the charged $l_{i}^{c}$ and neutral $\phi_{m}$ are singlets of $\mathrm{SU}(5)$. The couplings $\lambda_{1,2,3}$ give masses to the charge $-1 / 3$ quarks, charge $2 / 3$ quarks and charged leptons respectively, the couplings $\lambda_{4,5}$ separate naturally the light doublet and heavy triplet components of the $\mathbf{5}$ and $\overline{\mathbf{5}}$ Higgs multiplets, the coupling $\lambda_{6}$ plays an important role in the neutrino mass matrix as we shall see shortly, and the couplings $\lambda_{7,8}$ need not concern us here. In addition, the neutrino mass matrix involves a crucial non-renormalizable $F_{i} F_{j} H H$ coupling $\lambda_{9_{i j}}$ [15], as we shall also see shortly.

Prior to the realization of the importance of non-perturbative electroweak interactions in connection with the baryon asymmetry, we described several possibilities for the production of a net baryon number in the context of flipped $S U(5)$ [19. The alternatives depended on 1) the field content and 2) reheating subsequent to inflation. In the minimal flipped $S U(5)$ model [11, there are a limited number of fields whose decays can be used to produce a net baryon number. This has been the chief obstacle to writing down a satisfactory model of baryogenesis. Moreover, the breaking of flipped $S U(5)$ is accompanied by entropy production [21] which further impedes a large asymmetry, though we show below that the entropy production was previously overestimated.

Examining the field content of the flipped $S U(5)$ model above, the most obvious candidates to produce a baryon asymmetry would be the decays of color triplets in the $h$ and $\bar{h}$ multiplets. But it is well known [22] that one can not generate an asymmetry with a single $h, \bar{h}$ pair. Moreover, it is not possible to add additional Higgs representations to this model without defeating the natural doublet-triplet splitting mechanism [19]: additional $h, \bar{h}$ fields would require additional $H, \bar{H}$ fields, and these would give rise to light "uneaten" baryon-number-violating $u_{H}, d_{H}$ fields after GUT symmetry breaking, unless further modifications to the model are employed. Here, we will stick to the minimal model. As an alternative, one might also consider the massive $\nu_{H}^{c}, \nu_{\bar{H}}^{c}$ combination orthogonal to the flaton $\Phi$, discussed below, as a possible source of baryon asymmetry, but the decay of such a field (which is through the $h$ or $\bar{h}$ field) is always CP-conserving. We were therefore only left with the massive $\phi_{m}$ fields, which combine with $\nu_{i}^{c}$ to form massive Dirac states through the see-saw process. The only possibility for a net baryon number, therefore, appeared [19] to be the four-body decay channel $\phi \rightarrow F F F \bar{f}$. As this decay channel is heavily suppressed relative to the dominant two-body channels, a sizeable asymmetry was not a hopeful prospect.

Moreover, the final baryon asymmetry in any model with an "intermediate" scale is 
in general suppressed if the gauge symmetry is broken along a flat direction [23]. In flipped $S U(5), S U(5) \times U(1)$ is broken down to $S U(3) \times S U(2) \times U(1)$ by the F- and D- flat direction $\langle H\rangle=\langle\bar{H}\rangle \neq 0$. Because of the flatness, which is removed only by supersymmetry breaking, the gauge is symmetry is broken late by the flaton $\Phi$, the linear combination of $\nu_{H}^{c}$ and $\nu_{\bar{H}}^{c}$ in $H$ and $\bar{H}$ that develops a vev, and this is generally accompanied by the production of entropy [21]. Typically, the avoidance of excessive entropy production sets stringent constraints on the vev associated with the flat direction [24].

Inflation plays an important role in that the amount of entropy produced by flaton decays depends heavily on whether or not the gauge symmetry is restored subsequent to inflationary reheating. Previously we found that a sufficient baryon asymmetry could only be produced in the minimal flipped $S U(5)$ model in the strong reheating scenario [19, i.e. the one in which the gauge symmetry was restored. Even then the outcome was only marginal. In the weak reheating scenario, which as we emphasize below is the more natural possibility, the branching ratio to the four-body $\Delta B \neq 0$ channel was far too small to be significant [19].

In what follows, we present a simple mechanism to generate the baryon asymmetry based on the idea of Fukugita and Yanagida [14, which generates a lepton asymmetry that is then recycled by sphaleron effects to produce $\Delta B \neq 0$. Indeed, the previous scenario $(\phi \rightarrow F F F \bar{f})$ generates no net $B-L$, in which case sphaleron effects wipe out any $\Delta B=\Delta L \neq 0[$ [)

The generation of a net lepton asymmetry in flipped $S U(5)$ is closely related to the see-saw mechanism generating neutrino masses. Thus there may be a close connection between the solar neutrino problem and the baryon asymmetry. From the flipped superpotential above (1) one can easily write down the neutrino mass matrix 12

$$
\left(\nu_{i}, \nu_{i}^{c}, \phi_{i}\right) \quad\left(\begin{array}{ccc}
0 & m_{u} & 0 \\
m_{u} & \lambda_{9 i j} \frac{V^{2}}{M_{n r}} & \lambda_{6 i j} V \\
0 & \lambda_{6 i j} V & \mu_{i j}
\end{array}\right)\left(\begin{array}{c}
\nu_{i} \\
\nu_{i}^{c} \\
\phi_{j}
\end{array}\right)
$$

where $m_{u}=\lambda_{2 i j} v$ is the up-quark mass matrix, $V=\langle H\rangle=\langle\bar{H}\rangle \sim 10^{15} \mathrm{GeV}$ is the vacuum expectation value of the Higgs $\mathbf{1 0}$ and $\overline{\mathbf{1 0}}$ breaking $S U(5) \times U(1), \mu \simeq \lambda_{8}\left\langle\phi_{0}\right\rangle \simeq 10^{17} \mathrm{GeV}$, where $\left\langle\phi_{0}\right\rangle$ is the vev of one of the singlet fields, and is responsible for giving large masses to the other singlets. (Note there is still a singlet combination with a small vev which provides the necessary $h, \bar{h}$ mixing.) Finally, we include a mass term from a nonrenormalizable piece of the superpotential [15]

$$
\mathcal{L} \ni \lambda_{9 i j} \frac{1}{M_{n r}} F_{i} F_{j} \bar{H} \bar{H} \rightarrow \lambda_{q i j} \frac{V^{2}}{M_{n r}} \nu_{i}^{c} \nu_{j}^{c}
$$

Noting the hierarchy $m_{u} \ll \lambda_{9} \frac{V^{2}}{M_{n r}} \ll V \ll \mu$, we can identify the principal mass eigenvalues and eigenstates. There are states which are predominantly $\phi_{i}$ with masses $\mu$, and there is a set of states which are predominantly $\nu_{i}^{c}$ with masses $\lambda^{2}{ }_{6 i j} \frac{V^{2}}{\mu}$. The light neutrino masses are just $m_{\nu} \simeq \frac{m_{u}^{2} \mu}{\left(\lambda_{6}^{2} V^{2}\right)}\left(\right.$ when $\left.\lambda_{9} \mu \ll \lambda_{6}^{2} M_{n r}\right)$ :

$$
\left(m_{\phi+\ldots}\right)_{i j} \simeq \mu_{i j} ; \quad\left(m_{\nu^{c}+\ldots}\right)_{i j} \simeq \lambda_{6 i j}^{2} \frac{V^{2}}{\mu}, \quad\left(m_{\nu+\ldots}\right)_{i j} \simeq\left(\frac{m_{u}^{2} \mu}{\lambda_{6}^{2} V^{2}}\right)_{i j}
$$

1) Unless some of the lepton asymmetry can be stored in a weakly coupled field such as $e_{R}[7]$. 
Given the additional hierarchy in the up-quark mass matrix $\lambda_{21}: \lambda_{22}: \lambda_{23}=10^{-4}$ : $10^{-2}: 1$, and $\lambda_{6} \simeq \frac{1}{3}$, one finds for the three light neutrino masses $\left(m_{\nu e}, m_{\nu \mu}, m_{\nu \tau}\right) \sim$ $\left(10^{-7} \mathrm{eV}, 10^{-3} \mathrm{eV}, 10 \mathrm{eV}\right)$. Furthermore, the expected mixing between $\nu_{e}$ and $\nu_{\mu}$ is $\sin ^{2} 2 \theta_{e \mu} \sim$ $\frac{m_{u}}{m_{c}} \sim 10^{-2}$ for $\Delta m^{2} \sim 10^{-6}$. The fact that $m_{\nu \tau} \sim 10 \mathrm{eV}$ implies that this pattern of neutrino masses can simultaneously provide cosmological hot dark matter with $\Omega_{H D M}=$ $\Omega_{\nu \tau} \simeq 0.3$, as suggested by the COBE and other observations of large-scale structure, and give $\nu_{e}-\nu_{\mu}$ mixing sufficient to solve the solar neutrino problem [12]. We now show that the same neutrino mass matrix can also be used to account for the primordial baryon asymmetry. To generate a net baryon asymmetry, we first show that this model generates a net lepton asymmetry, which subsequently is transformed into a baryon asymmetry via sphaleron reprocessing, as in the original work of Fukugika and Yanagida [14]. In the FY mechanism, the CP-non-conserving out-of-equilibrium decays of a heavy right-handed neutrino into ordinary light left-handed leptons (and Higgses) produce a net lepton asymmetry much as the out-of-equilibrium decays of super-heavy gauge or Higgs bosons were originally used to generate a net baryon asymmetry [25].

The rate of decay of $\nu^{c} \rightarrow L+h$ is given by $\Gamma_{D} \simeq\left(\frac{\lambda_{2}^{2}}{16 \pi}\right) m_{\nu^{c}}=\frac{m_{u}^{2}}{16 \pi v^{2}} \lambda_{6}^{2} \frac{V^{2}}{\mu}$ where $\lambda_{2}=\frac{m_{u}}{v}$ is the up-quark Yukawa coupling. Decays will occur out of equilibrium if $\Gamma_{D}$ is smaller than the expansion ratio of the Universe $H$ at $T=m_{\nu^{c}}$ or $\left(\frac{\lambda_{2}^{2}}{16 \pi}\right) M_{P}<m_{\nu^{c}}$. In our case with $m_{\nu^{c}} \sim 10^{12} \mathrm{GeV}$, this requirement becomes $\lambda_{2}^{2} \lesssim 10^{-6}$, which is certainly true for first-generation Yukawa couplings. The final baryon asymmetry after sphaleron processing will be $n_{B} \sim n_{L} \sim \epsilon n_{\gamma}$ and $\frac{n_{B}}{s} \sim 10^{-2} \epsilon$, where $\epsilon$ is the CP asymmetry in the decay. (For the masses and couplings considered here, dimension-five operators of the form $\left(\frac{\lambda_{2}^{2}}{M_{\nu^{c}}}\right) \nu^{c} \nu^{c} h h$ will not erase the baryo and lepton asymmetries [26].)

In a cosmological model with inflation, COBE observations of the magnitude of the quadropole density fluctuations fix the overall inflationary scale 27, 20. The apparent COBE discovery of primordial microwave background fluctuations at the level $\frac{\delta \rho}{\rho} \simeq 5 \times$ $10^{-6}$ [9] fixes the inflationary scale and hence the inflaton mass [20]

$$
m_{\eta} \sim f e w \times 10^{11} \mathrm{GeV}
$$

and the reheat temperature to be $T_{R} \simeq 10^{8} \mathrm{GeV}$ in a generic inflationary model. The production of a lepton asymmetry by $\nu^{c}$ decay, therefore, requires $m_{\nu^{c}} \lesssim m_{\eta} \sim f e w \times$ $10^{11} \mathrm{GeV}$. We note that with a slight generation dependence in $\lambda_{6}, \frac{\lambda_{6_{1}}^{2}}{\lambda_{6_{3}}^{2}}=\frac{1}{10}$, we have $m_{\nu_{1}^{c}} \simeq 10^{11} \mathrm{GeV}$. The final baryon asymmetry is now given by 20.

$$
\frac{n_{B}}{s} \sim \frac{n_{L}}{s} \sim\left(\frac{m_{\eta}}{M_{P}}\right)^{\frac{1}{2}} \epsilon
$$

As we noted earlier, the baryon (lepton) asymmetry will be diluted by the entropy produced during the breaking of $S U(5) \times U(1)$ as $\Phi$ picks up its vev [21. This dilution factor, $\Delta$, is just the ratio of the entropy at the time of $\Phi$ decay to the entropy at the time of inflaton decay (i.e., the entropy in eq. (6) appropriately scaled),

$$
\Delta=\frac{s\left(R_{d \Phi}\right)}{s\left(R_{d \eta}\right)}\left(\frac{R_{d \Phi}}{R_{d \eta}}\right)^{3}=\frac{\alpha_{\Phi}{ }^{3 / 2} \tilde{m}^{9 / 2} M_{P}^{3 / 2}}{V^{3} T_{R}^{3}}\left(\frac{R_{d \Phi}}{R_{d \eta}}\right)^{3}=\frac{V^{3} m_{\eta}^{3 / 2}}{\alpha_{\Phi}{ }^{1 / 2} \tilde{m}^{3 / 2} M_{P}^{3}} \sim 10^{3}
$$


where we have taken the flaton decay rate to be $\Gamma_{\Phi}=\alpha_{\Phi} \frac{\tilde{m}^{3}}{V^{2}}, \alpha_{\Phi} \sim 10^{-3}$, the susy breaking scale to be $\tilde{m} \sim 10^{-16} M_{P}, T_{R}=\frac{m_{\eta}^{3 / 2}}{M_{P}^{1 / 2}}, R_{d \eta(\Phi)}$ is the cosmological scale factor at the time of inflaton (flaton) decay (see refs [24, 27, 21] for further details),

$$
\begin{aligned}
s\left(R_{d \Phi}\right)=\left[\rho\left(R_{d \Phi}\right)\right]^{3 / 4} & =\frac{\alpha_{\Phi}{ }^{3 / 2} \tilde{m}^{9 / 2} M_{P}^{3 / 2}}{V^{3}} \\
s\left(R_{d \eta}\right) & =\left[\rho\left(R_{d \eta}\right)\right]^{3 / 4}=T_{R}^{3}
\end{aligned}
$$

and

$$
\left(\frac{R_{d \Phi}}{R_{d \eta}}\right)=\frac{m_{\eta}^{2} V^{2}}{\alpha_{\Phi}^{2 / 3} \tilde{m} M_{P}^{2}}
$$

The final baryon-to-entropy ratio becomes

$$
\frac{n_{B}}{s}=\left(\frac{m_{\eta}}{M_{P}}\right)^{\frac{1}{2}} \epsilon \frac{1}{\Delta}=\frac{\epsilon \alpha_{\Phi}^{1 / 2} \tilde{m}^{3 / 2} M_{P}^{5 / 2}}{V^{3} m_{\eta}}
$$

and we now estimate $\epsilon$.

When the conjugate neutrinos $\nu^{c}, \overline{\nu^{c}}$ decay, a difference between the branching ratios $\nu^{c} \rightarrow L+h, \overline{\nu^{c}} \rightarrow \bar{L}+\bar{h}$ appears if $\mathrm{CP}$ is violated. The dominant contribution to this comes from the interference of the two diagrams shown in the figure, namely the tree-level diagram and the one-Higgs-loop radiative correction [14]:

$$
\epsilon=\left(\frac{9}{4 \pi}\right) \frac{\operatorname{Im}\left(\lambda_{2 i j} \lambda^{\dagger}{ }_{2 j k} \lambda^{\dagger}{ }_{2 k l} \lambda_{2 l i}\right) I\left(\frac{M_{j}^{2}}{M_{i}^{2}}\right)}{\lambda_{2 i j} \lambda^{\dagger}{ }_{2 i j}}
$$

where

$$
I(x) \equiv x^{\frac{1}{2}}\left[1+(1+x) \ln \left(\frac{x}{(1+x)}\right)\right]
$$

For reasons that will soon become apparent, we expect the dominant contribution to come from third-generation particles in the decays of the first-generation $\nu^{c}$ and $\bar{\nu}^{c}$ :

$$
\epsilon_{13}=\frac{9}{8 \pi}\left|\lambda_{233}\right|^{2}\left(\frac{M_{1}}{M_{3}}\right) \delta
$$

where $\delta$ is the CP-violating phase factor and we have assumed that $\left|\lambda_{211}\right|,\left|\lambda_{212}\right| \ll\left|\lambda_{213}\right|$. Since the see-saw light neutrino masses are expected to be in the hierarchy $m_{\nu_{\tau}}\left(\propto \frac{\left|\lambda_{233}\right|^{2}}{\left.m_{\nu_{1}^{c}}\right)} \gg\right.$ $m_{\nu_{\mu}}\left(\propto \frac{\left|\lambda_{222}\right|^{2}}{m_{\nu_{2}^{c}}}\right) \gg m_{\nu_{?} ?}\left(\propto \frac{\left|\lambda_{211}\right|^{2}}{m_{\nu_{3}^{c}}}\right)$, we see that $\left|\epsilon_{13}\right| \gg\left|\epsilon_{12}\right|>\left|\epsilon_{11}\right|$. The corresponding value of the present-day baryon-to-entropy ratio is now

$$
\frac{n_{B}}{n_{\gamma}} \simeq \epsilon\left(\frac{m_{\eta}}{M_{P}}\right)^{\frac{1}{2}} \frac{1}{\Delta} \simeq \frac{9}{8 \pi}\left|\lambda_{233}\right|^{2}\left(\frac{m_{\nu_{1}^{c}}}{m_{\nu_{3}^{c}}}\right)\left(\frac{m_{\eta}}{M_{P}}\right)^{\frac{1}{2}} \frac{\delta}{\Delta}
$$

Or, in terms of the magnitude value of density fluctuations measured by COBE, we can write

$$
\frac{n_{B}}{n_{\gamma}} \simeq \frac{9}{80 \pi}\left|\lambda_{233}\right|^{2}\left(\frac{m_{\nu_{1}^{c}}}{m_{\nu_{3}^{c}}}\right) \sqrt{\frac{\delta \rho}{\rho}} \frac{\delta}{\Delta}
$$


Recent theoretical, experimental and observational developments enable us to put in numbers for most of the factors in the last formula. The value of $\left|\lambda_{233}\right|$ can be estimated from indirect determinations [28] of $m_{t}$ via electroweak radiative corrections $\left(m_{1} 24_{-28}^{+26} \mathrm{GeV}\right)$ or two irresponsible theorists' interpretation 29 o one published event $\left(m_{t}=131_{-11}^{+22} G e V\right)$, and the relation

$$
\left|\lambda_{2_{33}}\left(m_{t}\right)\right|=\frac{g m_{t}}{2 M_{W} \sin \beta}
$$

where $g$ is the $S U(2)$ gauge coupling at the electroweak scale and $\tan \beta=\frac{v_{2}}{v_{1}}$ is the ratio of supersymmetric Higgs vev's. The latter is believed to be larger than unity, so that $\frac{1}{\sqrt{2}}<\sin \beta$ We therefore estimate that

$$
\frac{g}{\sqrt{2}} \simeq\left|\lambda_{233}\left(m_{t}\right)\right| \simeq g
$$

However, the effective renormalization scale in the CP-violating $\nu^{c} / \bar{\nu}^{c}$ decays is much larger, and $h_{33}\left(m_{\nu^{c}}\right)$ may be somewhat smaller, so we take

$$
h_{33}\left(m_{\nu^{c}}\right) \simeq \frac{g}{\sqrt{2}}
$$

as our central value. This coincides with the value calculated form first principles in versions of flipped $S U(5)$ derived from string theory, assuming plausible mixing between the $t$ quark and other charge $\frac{2}{3}$ fields.

The values of the ratios $\left(\frac{M_{1}}{M_{2,3}}\right)$ were discussed in ref. [12]. We estimated there that $\left(\frac{m_{\nu_{2}^{c}}}{m_{\nu_{1}^{c}}}\right)=0(1)$ on the basis of the known values of $m_{u}$ and $m_{c}$ and the value of the $\nu_{e}-\nu_{\mu}$ mixing angle that best fits the solar neutrino data, assuming the MSW mechanism. We also found that the best fit to the combination of COBE and other data on primordial density perturbations, which prefer an admixture of about $30 \%$ hot dark matter for which the best (only?) candidate is a $\nu_{\tau}$ weighing $0(10) \mathrm{eV}$, would be with $\frac{m_{\nu_{3}^{c}}}{m_{\nu_{2}^{c}}}=0(10)$. Therefore we put $\frac{m_{\nu_{1}^{c}}}{m_{\nu_{3}^{c}}} \simeq \frac{1}{10}$ in equation $(16)$.

The only remaining unknown is the CP-violating phase factor $\delta$ in (16) and we estimate

$$
\frac{n_{B}}{n_{\gamma}} \simeq 2 \times 10^{-6} \frac{\delta}{\Delta} \simeq 2 \times 10^{-9} \delta
$$

We conclude that for plausible values of $\delta$, this mechanism is completely consistent with the value $\frac{n_{B}}{n_{\gamma}} \sim 3 \times 10^{-10}$ extracted from the concordance of data on primordial nucleosynthesis [30], even with the possibility of some extra entropy generation subsequent to the $\nu^{c}$ and $\bar{\nu}^{c}$ decays.

We find it remarkable that the simplest flipped SU(5) model, in addition to all its other well-documented virtues and its motivation from string theory, also ties together in a very economical way baryogenesis, solar neutrino physics, COBE and Dark Matter. The same flipped heavy neutrino that plays a key role in the see-saw mass matrix, accommodating the MSW interpretation of the apparent solar neutrino deficit and the apparent preference for an admixture of hot Dark Matter, also generates a lepton (and hence, thanks to sphalerons, a baryon) asymmetry in a very simple and convincing way. 
Many of the parameters needed to make a quantitative estimate of the resulting baryonto-entropy ratio have recently been fixed or severely constrained by accelerator, GALLEX and COBE data. Furthermore, as we have emphasized previously [12], these astrophysical aspects of the flipped $\mathrm{SU}(5)$ model ca in principle soon be tested via accelerator experiments searching for $\nu_{\mu}-\nu_{\tau}$ oscillations.

\section{Acknowledgements}

The work of DVN was supported in part by DOE grant DE-FG05-91-ER-40633 and by a grant from Conoco Inc. The work of KAO was supported in part by DOE grant DEAC02-83ER-40105, and by a Presidential Young Investigator Award. 


\section{REFERENCES}

[1] G. 't Hooft, Phys. Rev. Lett. 37(1976) 8; V. Kuzmin, V. Rubakov and M. Shaposhnikov, Phys.Lett. B155 (1985) 36

[2] P. Arnold and L. McLerran, Phys.Rev. D36 (1987) 581, Phys.Rev. D37 (1988) 1020.

[3] M.E. Shaposhnikov, Nucl. Phys. B287(1987)757; B299(1988)797; A.I. Bochkarev, V. Kuzmin and M.E. Shaposhnikov, Phys. Lett. B244(1990)275; A.I. Bochkarev, S.Yu. Khlebnikov and M.E. Shaposhnikov, Nucl. Phys. B329(1990)490; L. McLerran, Phys. Rev. Lett. 62(1989)1075; N. Turok and J. Zadrozny, Phys. Rev. Lett. 65(1990)2331; Nucl. Phys. B358(1991)471; L. McLerran, M.E. Shaposhnikov, N. Turok and M. Voloshin, Phys. Lett. B256(1991)251; A. Cohen, D. Kaplan and A. Nelson, Nucl. Phys. B349(1991)727; Phys. Lett. B263(1991)86; G.W. Anderson and L.J. Hall, Phys. Rev. D45(1992)2685; M. Dine, P. Huet, R.S. Singleton Jr. and L. Susskind, Phys. Lett. B257(1991)351;M. Dine, P. Huet and R.S. Singleton Jr., Nucl. Phys. B375(1992)625; M. Dine, R.G. Leigh, P. Huet, A. Linde and D. Linde, SLAC preprints PUB-5740 and PUB-5741 (1992).

[4] J.N. Bahcall and R.N. Ulrich, Rev. Mod. Phys. 60(1988) 297.

[5] S.D.M. White, C.S. Frenk and M. Davis, Ap.J. 274(1983) 61.

[6] V.A. Kuzmin, V.A. Rubakov and M.E. Shaposhnikov, Phys. Lett. B191 (1987) 171; A.E. Nelson and S.M. Barr, Phys. Lett. B246 (1990)141; H. Dreiner and G.G. Ross, Oxford preprint OUTP-92-08P (1992).

[7] B. Campbell, S. Davidson, J. Ellis and K. A. Olive, CERN preprint TH.6642/92 (1992), Phys. Lett. B in press (1992).

[8] GALLEX Collaboration, P. Anselmann et al.,Phys. Lett. B285 (1992) 390.

[9] F.L. Wright et al., Ap. J. Lett. 396 (1992) L13.

[10] S. Barr, Phys. Lett. B112 (1982) 219; Phys. Rev. D40 (1989) 2457; J.-P. Derendinger, J. Kim and D.V. Nanopoulos, Phys. Lett. B139(1984)170.

[11] I. Antoniadis, J. Ellis, J. Hagelin and D.V. Nanopoulos, Phys. Lett. B194(1987) 231.

[12] J. Ellis, J.L. Lopez and D.V. Nanopoulos, Phys. Lett. B292 (1 189.

[13] L.Wolfenstein, Phys. Rev. D17 (1978) 2369; D20 (1979) 2634; S.P. Mikheyev and A. Yu. Smirnov, Yad. Fiz. 42 (1985) 14 Nuov. Cim. 9C (1986) 17.

[14] M. Fukugita and T. Yanagida, Phys.Lett. B174 (1986) 45.

[15] I. Antoniadis, J. Ellis, J. Hagelin and D.V. Nanopoulos, Phys. Lett. B208(1988) 209.

[16] G. Leontaris, Phys. Lett. B207 (1988) 447; G. Leontaris and D.V. Nanopoulos, Phys. Lett. B212 (1988) 327; G. Leontaris and K. Tamvakis, Phys. Lett. B224 (1989) 319; S. Abel, Phys. Lett. B234 (1990) 113; I. Antoniadis, J. Rizos and K. Tamvakis, Phys. Lett. B279 (1992) 281.

[17] J.L. Lopez and D.V. Nanopoulos, Phys. Lett. B268 (1991) 359.

[18] R. Barbieri, D.V. Nanopoulos and A. Masiero, Phys. Lett. B98 (1981) 191; A. Masiero, D.V. Nanopoulos, K. Tamvakis and T. Yanagida, Z. Phys. C17 (1983) 33.

[19] B. Campbell, J. Ellis, J. Hagelin, D.V. Nanopoulos and K.A. Oliv Phys. Lett. B200 (1988) 483; J. Ellis, J.Hagelin, D.V. Nanopoulos, and K.A. Olive, Phys. Lett. B207(1988) 451.

[20] B. Campbell, S. Davi and K. A. Olive, University of Minnesota preprint UMN-TH1114/92 (1992). 
[21] B. Campbell, J. Ellis, J.Hagelin, D.V. Nanopoulos and K.A. Olive Phys. Lett. B197 (1987) 355.

[22] D.V. Nanopoulos and S. Weinberg, Phys. Rev. D20 (1979) 2484.

[23] K. Yamamoto, Phys. Lett. B168 (1986) 341; K. Enqvist, D.V. Nanopoulos and M. Quiros, Phys. Lett. B169 (1986) 343.

[24] J. Ellis, K. Enqvist, D.V. Nanopoulos and K.A. Olive, Phys. Lett. B188 (1987) 415.

[25] S. Weinberg, Phys. Rev. Lett. 42 (1979) 850; D. Toussaint, S. B. Treiman, F. Wilczek and A. Zee, Phys. Rev. D19 (1979) 1036;J. Ellis, M.K. Gaillard and D.V. Nanopoulos, Phys. Lett. B80 (1979) 3

[26] M. Fukugita and F. Yanagida, Phys.Rev. D42 (1990) 1285; J.A. Harvey and M.S. Turner, Phys.Rev. D42 (1990) 3344; B.A. Campbell, S. Davidson, J. Ellis and K.A. Olive, Phys.Lett. B256 (1991 457; CERN preprint TH.6208/91 (1991), Astropart.Phys. 1 (1992) (in press) Fischler, G.F. Giudice, R.G. Leigh and S. Paban, Phys.Lett. B258 (1991) 45

[27] J. Ellis, K. Enqvist, D.V. Nanopoulos and K.A. Olive, Phys. Lett. B191(1987) 343.

[28] J. Ellis, G.L. Fogli and E. Lisi, CERN preprint TH.6568/92 (1992).

[29] R.H. Dalitz and G.R. Goldstein, Phys. Lett. B287 (1992) 225.

[30] T. Walker, D.N. Schramm, G. Steigman, K.A. Olive and K. Kang, Ap. 376 (1991) 51. 


\section{Figure caption}

The tree and one-Higgs-loop diagrams whose interference gives the largest contribution to the lepton asymmetry in heavy singlet neutrino decay in flipped SU(5). 